\title{
Analytical models of axisymmetric reaction-diffusion phenomena in composite media
}

\author{
Robert A. Zimmerman ${ }^{\mathrm{a}, \mathrm{b}}$, Todd A. Jankowski ${ }^{\mathrm{b}}$, Daniel M. Tartakovsky ${ }^{\mathrm{a}}$ \\ ${ }^{a}$ Department of Mechanical and Aerospace Engineering, University of California, San Diego, 9500 Gilman Drive, La \\ Jolla, CA 92093, United States \\ ${ }^{b}$ Applied Engineering Technology-1, Los Alamos National Laboratory, Los Alamos, NM 87545, United States
}

\begin{abstract}
Reaction-diffusion equations describe a number of physical, chemical, and biological phenomena, many of which occur in composite environments with piece-wise constant diffusion coefficients. We develop semi-analytical solutions of axisymmetric reaction-diffusion equations with first-order reaction kinetics and continuous transient boundary conditions. These solutions are directly applicable to heat conduction in composite media with transient boundary conditions and heat generation. The solutions lose their robustness in the long time regime, when the Laplace variable tends to zero. This limitation is overcome by the use of corresponding steady-state solutions.
\end{abstract}

Keywords: Heat conduction, Diffusion, Reaction, Composite media, Laplace transform

\section{Introduction}

Transient one-dimensional solutions for heat conduction in composite media have been developed by several authors. Ozisik [10] provides an extensive review of various solutions obtained via Laplace transformations and Green's functions to handle transient problems, and the generalized orthogonal expansion technique to solve homogeneous or steady state problems. Carslaw and Jaeger [3] present solutions derived by means of the Laplace transformation. Sun [11] and de Monte [9] derive solutions utilizing the eigenfunction expansion method. Huang [6] uses Green's functions to derive solutions for periodic boundary conditions. Dias [5] presents a recursive method based on Green's functions to develop a solution. Beck et. al [2] develop a Galerkin-based Green's function method to analyze multidimensional problems. Aviles-Ramos et. al [1] develop a two-layer composite heat conduction solution with periodic boundary conditions using a spectral method with an orthotropic layer to estimate thermophysical properties.

This work employs Laplace transforms to improve previous solutions by solving problems with first-order reaction and source terms in composite media with any number of layers, subject to transient boundary conditions. The first-order reaction term in mass transfer problems is equivalent to the heat generation term in heat conduction problems. Transient boundary conditions of the periodic form are of particular relevance to many problems such as seasonal or diurnal temperature changes outside of a structure. Another applica- tion is diffusion through fractured rock [8]. The model developed here can be incorporated as a multi-layered rock medium in the fracture matrix.

More recent work by Sun et. al [11] and de Monte [9] uses eigenfunction expansions to derive nominally analytical solutions, even though the eigenvalues must be calculated numerically and the accuracy of the solution is improved by including more terms in the eigenvalue calculation. The number of required terms in the eigenfunction expansion method increases for small times [11]. The eigenfunction method is advantageous for large-time solutions, as Laplace transform solutions suffer numerical round off for large time values. We overcome this disadvantage by implementing the final value theorem, which produces an exact steady-state solution in real space with no numerical calculation and no need for eigenvalue calculation or increased term inclusion for accuracy. Thus the solution presented here is both valid and accurate for all times. The only exception to this is some intermediate-time regimes characterized by large Damköhler numbers.

A unique advantage of the Laplace transform methods, as it is applied to the current problem, is that the boundary conditions can be isolated as a multiple of the rest of the solution rather than as a component tied inside of an integral. This unique feature allows for various types of boundary conditions to be easily incorporated into the problem. Isolation of the boundary conditions is also useful for linking multiple solutions together 
in order to create a composite solution. The difficulty often presented by the Laplace transform method is the transformation back to real space. Though this inverse transformation can be done for each and every potential boundary condition, this can be a tedious task in systems with a large number of layers. Li et. al [7] presented an analytical solution for a first-order reaction-diffusion problem in a two layer slab, which does not consider arbitrary time-dependent boundary conditions. Extension of this solution to an $m$-layered solution (where $m$ is any number of layers) is cumbersome. Our method relies on the numerical inverse Laplace transform algorithm developed by De Hoog [4] to handle the $m$-layered solution. Though this presents a numerical finale to the solution, it yields a very quick and accurate solution for a wide range of problems.

\section{Methodology}

\subsection{The Model}

The physical system models used in this paper are layered pieces of material. For cylindrical and spherical coordinate problems the layers are concentric hollow circles or spheres. Figure 1 depicts the problem in cylindrical coordinates. Each layer is numbered and has its own geometric and physical properties. The solution is robust enough to handle different layer thicknesses, as well as varying diffusion and reaction coefficients and source terms.

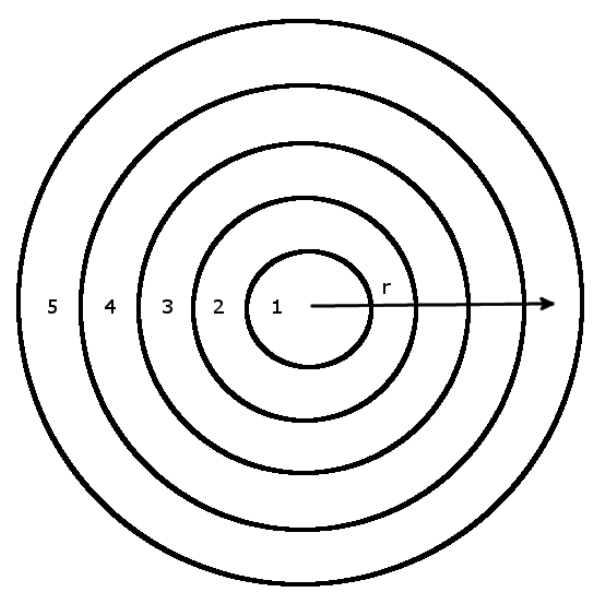

Figure 1: A radial layered model.

A first-order reaction-diffusion equation is based on Fick's law with a first-order reaction term,

$$
\frac{\partial \mathcal{C}}{\partial t}=\mathcal{D} \nabla^{2} \mathcal{C}+(\mathcal{R}+\Phi) \mathcal{C}
$$

where $\mathcal{D}$ is the diffusion coefficient, $\mathcal{R}$ is the firstorder reaction rate coefficient, and $\Phi$ is an un- known source term that is constant with respect to time and space.

\subsection{Non-Dimensional Form}

The governing equation is transformed into its non-dimensional form by defining the nondimensional terms as

$$
\begin{aligned}
C=\frac{\mathcal{C}}{\mathcal{C}_{c}}, & D=\frac{\mathcal{D}}{\mathcal{D}_{c}}, \quad R=\frac{\mathcal{R}}{\mathcal{R}_{c}}, \quad \psi=\frac{\Phi}{\mathcal{R}_{c}} \\
\xi=\frac{x}{L_{c}}, & \rho=\frac{r}{L_{c}}, \quad \tau=\frac{t \mathcal{D}_{c}}{L_{c}^{2}}
\end{aligned}
$$

where the subscript $c$ denotes the characteristic value of the relevant quantity. This transforms (1) into

$$
\frac{\partial C}{\partial \tau}=D \nabla^{2} C+D a(R+\psi) C
$$

where $D a$ is the Damköhler number defined as

$$
D a=\frac{\mathcal{R}_{c} L_{c}^{2}}{\mathcal{D}_{c}}
$$

and $\mathcal{R}_{c}$ and $\mathcal{D}_{c}$ are the largest coefficients in the composite medium, such that

$$
0 \leq D \leq 1, \quad 0 \leq R \leq 1, \quad 0 \leq \psi \leq 1 .
$$

\subsection{Laplace Transform}

The Laplace transform is defined as

$$
\hat{f}(s)=\mathcal{L}(f(\tau))=\int_{0}^{\infty} f(\tau) e^{-s \tau} \mathrm{d} \tau .
$$

It is used in this work for two primary reasons. First, it allows the boundary condition to be a multiplying factor of the equation describing the problem in Laplace space rather than being a component of an integral in the governing equation. Second, it significantly simplifies the derivation of the solution by reducing the dimensionality of a PDE. Since

$$
\hat{f}(s)=\mathcal{L}\left(\frac{\partial f(\tau)}{\partial \tau}\right)=s \hat{f}(s)-f(0),
$$

taking the Laplace transform of (3) yields

$$
s \hat{C}-C_{0}=\mathcal{L}\left(D \nabla^{2} C+D a(R+\psi) C\right)
$$

where $C_{0}=\mathcal{C}_{0} / \mathcal{C}_{c}$ is the initial condition.

The general Laplace space solutions in each coordinate system (Cartesian, cylindrical, and spherical, respectively) are

$$
\begin{aligned}
& \hat{C}=A e^{\beta \xi}+B e^{-\beta \xi}+C_{0} / \mathcal{S} \\
& \hat{C}=A I_{0}(\beta \rho)+B K_{0}(\beta \rho)+C_{0} / \mathcal{S} \\
& \hat{C}=A \frac{e^{-\beta \rho}}{\rho}+B \frac{e^{\beta \rho}}{\beta \rho}+\frac{C_{0}}{\mathcal{S}}
\end{aligned}
$$

where $\mathcal{S}=s-D a(R+\psi), \beta=\sqrt{\mathcal{S} / D}$ and $A$ and $B$ are constants of integration. 


\subsection{Special Cases}

There are three particular cases that are considered in this model. The first is referred to as the finite Neumann case; it has a flux (Neumann) boundary condition at one boundary and a Dirichlet boundary condition at the other. The second is the finite Dirichlet case, with a Dirichlet boundary condition at each boundary. The third is the semi-infinite Dirichlet case, which produces the same results regardless of whether a Neumann or Dirichlet boundary condition is used at infinity. The cases are described in more detail below. Let $a$ and $b$ be the boundaries of each layer and $\hat{\phi}$ denote the time-dependent boundary condition in the Laplace space. Then,

1 Finite Neumann, the solution in the range $a \leq \xi \leq b$ with boundary conditions

$$
\frac{\partial \hat{C}(a, s)}{\partial \xi}=\hat{\phi}_{a}(s), \quad \hat{C}(b, s)=\hat{\phi}_{b}(s) \text {. }
$$

2 Finite Dirichlet, the solution in the range $a \leq \xi \leq b$ with boundary conditions

$$
\hat{C}(a, s)=\hat{\phi}_{a}(s), \quad \hat{C}(b, s)=\hat{\phi}_{b}(s) .
$$

3 Semi-infinite Dirichlet, the solution in the range $a \leq \xi \leq \infty$ with the boundary conditions

$$
\begin{aligned}
\hat{C}(a, s) & =\hat{\phi}_{a}(s), \\
\hat{C}(\infty, s) & =\frac{C_{0}}{\mathcal{S}} \quad \text { or } \quad \frac{\partial \hat{C}(\infty, s)}{\partial \xi}=0 .
\end{aligned}
$$

In cylindrical and spherical coordinates the solid layer at the origin uses the finite Neumann case with $\hat{\phi}_{a}(s)=0$. Typically it is assumed that $a<b$ but this can be ignored in order to accomplish a wider breadth of possible combinations. For each coordinate system and each boundary condition case the integration constant $A(s)$ and $B(s)$ in (9)-(11) are given by

\section{Cartesian}

\section{Finite Neumann}

$$
\begin{aligned}
& A=\frac{\left(\hat{\phi}_{b}-\frac{C_{0}}{\mathcal{S}}\right) \mathcal{E}(2 a+b)+\frac{\hat{\phi}_{a}}{\beta} \mathcal{E}(a+2 b)}{\mathcal{E}(2 b)+\mathcal{E}(2 a)} \\
& B=\frac{\left(\hat{\phi}_{b}-\frac{C_{0}}{\mathcal{S}}\right) \mathcal{E}(b)-\frac{\hat{\phi}_{a}}{\beta} \mathcal{E}(a)}{\mathcal{E}(2 b)+\mathcal{E}(2 a)}
\end{aligned}
$$

where $\mathcal{E}(x) \equiv \exp (-\beta x)$.

2 Finite Dirichlet

$$
\begin{aligned}
& A=\frac{\hat{\phi}_{a}-\hat{\phi}_{b} \mathcal{E}(a-b)+\frac{C_{0}}{\mathcal{S}}[\mathcal{E}(a-b)-1]}{\mathcal{E}(-a)-\mathcal{E}(a-2 b)} \\
& B=\frac{\hat{\phi}_{b}-\hat{\phi}_{a} \mathcal{E}(a-b)+\frac{C_{0}}{\mathcal{S}}[\mathcal{E}(a-b)-1]}{\mathcal{E}(b)-\mathcal{E}(2 a-b)}
\end{aligned}
$$

3 Semi-infinite Dirichlet

$$
A=0, \quad B=\left(\hat{\phi}_{a}-\frac{C_{0}}{\mathcal{S}}\right) \mathcal{E}(-a) .
$$

\section{Cylindrical}

1 Finite Neumann

$$
\begin{aligned}
& A=\left(\hat{\phi}_{b}-\frac{C_{0}}{\mathcal{S}}\right) \frac{K_{1}(\beta a)}{\mathcal{B}_{N}}+\frac{\hat{\phi}_{a}}{\beta} \frac{K_{0}(\beta b)}{\mathcal{B}_{N}} \\
& B=\left(\hat{\phi}_{b}-\frac{C_{0}}{\mathcal{S}}\right) \frac{I_{1}(\beta a)}{\mathcal{B}_{N}}-\frac{\hat{\phi}_{a}}{\beta} \frac{I_{0}(\beta b)}{\mathcal{B}_{N}}
\end{aligned}
$$

where $\mathcal{B}_{N} \equiv K_{1}(\beta a) I_{0}(\beta b)+K_{0}(\beta b) I_{1}(\beta a)$.

2 Finite Dirichlet

$$
\begin{aligned}
A= & \left(\hat{\phi}_{b}-\frac{C_{0}}{\mathcal{S}}\right) \frac{K_{0}(\beta a)}{\mathcal{B}_{D}} \\
& -\left(\hat{\phi}_{a}-\frac{C_{0}}{\mathcal{S}}\right) \frac{K_{0}(\beta b)}{\mathcal{B}_{D}} \\
B= & \left(\hat{\phi}_{a}-\frac{C_{0}}{\mathcal{S}}\right) \frac{I_{0}(\beta b)}{\mathcal{B}_{D}} \\
& -\left(\hat{\phi}_{b}-\frac{C_{0}}{\mathcal{S}}\right) \frac{I_{0}(\beta a)}{\mathcal{B}_{D}}
\end{aligned}
$$

where $\mathcal{B}_{D} \equiv K_{0}(\beta a) I_{0}(\beta b)-K_{0}(\beta b) I_{0}(\beta a)$.

3 Semi-infinite Dirichlet

$$
A=0, \quad B=\frac{\left(\hat{\phi}_{a}-C_{0} / \mathcal{S}\right)}{K_{0}(\beta a)} .
$$

\section{Spherical}

\section{Finite Neumann}

$$
\begin{aligned}
A & =\frac{b\left(\hat{\phi}_{b}-\frac{C_{0}}{\mathcal{S}}\right)(\beta a-1) \mathcal{E}(b-a)-\hat{\phi}_{a} a^{2}}{(\beta a+1) \mathcal{E}(a)+(\beta a-1) \mathcal{E}(2 b-a)} \\
B & =\beta \frac{b\left(\hat{\phi}_{b}-\frac{C_{0}}{\mathcal{S}}\right)(\beta a+1)+\hat{\phi}_{a} a^{2} \mathcal{E}(b-a)}{(\beta a+1) \mathcal{E}(-b)+(\beta a-1) \mathcal{E}(b-2 a)} .
\end{aligned}
$$

\section{Finite Dirichlet}

$$
\begin{aligned}
A & =\frac{a\left(\hat{\phi}_{a}-\frac{C_{0}}{\mathcal{S}}\right)-b\left(\hat{\phi}_{b}-\frac{C_{0}}{\mathcal{S}}\right) \mathcal{E}(b-a)}{\mathcal{E}(a)-\mathcal{E}(2 b-a)} \\
B & =\beta \frac{b\left(\hat{\phi}_{b}-\frac{C_{0}}{\mathcal{S}}\right) \mathcal{E}(b)-a\left(\hat{\phi}_{a}-\frac{C_{0}}{\mathcal{S}}\right) \mathcal{E}(2 b-a)}{1-\mathcal{E}(2 b-2 a)}
\end{aligned}
$$

3 Semi-infinite Dirichlet

$$
A=a\left(\hat{\phi}_{a}-\frac{C_{0}}{\mathcal{S}}\right) \mathcal{E}(-a), \quad B=0
$$




\subsection{Final Value Theorem}

When the analytical inversion of our Laplacetransformed solutions becomes unfeasible, we employ the numerical inversion with the De Hoog algorithm [4]. This algorithm can introduce significant numerical round-off errors for small values of the Laplace variable $s$ or, equivalently, large values of time $\tau$. For $\tau \rightarrow \infty$, we use the final-value theorem,

$$
\lim _{\tau \rightarrow \infty} f(\tau)=\lim _{s \rightarrow 0} s \hat{f}(s)
$$

to obtain a steady-state solution without computing the inverse Laplace transform.

\subsection{Composite Solution}

A composite solution is developed by linking together the individual solutions (9)-(11). In order to connect the solution for each layer to the next the appropriate case is selected that best describes the boundary conditions for the layer(s) under consideration. Each layer's solution is then joined by enforcing the continuity of both concentration and mass flux across interfaces between layers. The continuity conditions for the $i$-th interface are

$$
\begin{aligned}
\hat{C}_{i}\left(b_{i}, s\right) & =\hat{C}_{i+1}\left(a_{i+1}, s\right) \\
D_{i} \frac{\partial \hat{C}_{i}\left(b_{i}, s\right)}{\partial \xi} & =D_{i+1} \frac{\partial \hat{C}_{i+1}\left(a_{i+1}, s\right)}{\partial \xi} .
\end{aligned}
$$

The problem is solved by developing a series of equations that describe the transport between two layers. This procedure comprises the following steps. First, a general solution $\hat{C}(\xi, s)$ for the appropriate coordinate system is chosen from (9)-(11) for the layers $i$ and $(i+1)$, $\hat{C}_{i}(\xi, s)$ and $\hat{C}_{i+1}(\xi, s)$; the interfaces $i=2, \ldots, \eta$ are treated as Dirichlet boundaries, with (yet unknown) boundary functions $\hat{\phi}_{i}(s)$ prescribed at the $i$-th interface. Second, boundary conditions (25) and (26) at the interface $\xi=b_{i}=a_{i+1}$ between the layers $i$ and $(i+1)$ are applied to $\hat{C}_{i}(\xi, s)$ and $\hat{C}_{i+1}(\xi, s)$. This yields an algebraic equation,

$$
A_{i} \hat{\phi}_{i}+B_{i} \hat{\phi}_{i+1}+F_{i+1} \hat{\phi}_{i+2}=E_{i}
$$

which relates the interfacial concentration $\hat{\phi}_{i+1}$ (at $\left.\xi=b_{i}=a_{i+1}\right)$ to its neighbors $\hat{\phi}_{i}\left(\right.$ at $\left.\xi=a_{i}\right)$ and $\hat{\phi}_{i+2}\left(\right.$ at $\left.\xi=b_{i+1}\right)$. The coefficients $A_{i}, B_{i}$, and $F_{i+1}$, as well as the free term $E_{i}$, are all functions of the Laplace variable $s$ and the spatial coordinate $\xi$. For $\eta$ layers, i.e., for $i=2, \ldots, \eta,(27)$ gives rise to a linear system of $\eta-1$ equations,

$$
\mathbf{H} \hat{\phi}=\mathbf{e}
$$

for $\eta-1$ unknowns $\hat{\phi}=\left(\hat{\phi}_{2}, \hat{\phi}_{3}, \ldots, \hat{\phi}_{\eta}\right)^{\top}$. Here $\mathbf{H}$ is a tridiagonal matrix

$$
\mathbf{H}=\left[\begin{array}{ccccccc}
B_{1} & F_{2} & 0 & 0 & 0 & \cdots & 0 \\
A_{2} & B_{2} & F_{3} & 0 & 0 & \cdots & 0 \\
0 & A_{3} & B_{3} & F_{4} & 0 & \cdots & 0 \\
0 & 0 & A_{4} & B_{4} & F_{5} & \cdots & 0 \\
\vdots & \vdots & & \ddots & \ddots & \ddots & \vdots \\
0 & 0 & 0 & \cdots & A_{\eta-2} & B_{\eta-2} & F_{\eta-1} \\
0 & 0 & 0 & \cdots & 0 & A_{\eta-1} & B_{\eta-1}
\end{array}\right],
$$

and the vector e has components

$$
\mathbf{e}=\left(E_{1}-A_{1} \hat{\phi}_{1}, E_{2}, E_{3}, \ldots, E_{\eta-1}-F_{\eta} \hat{\phi}_{\eta+1}\right)^{\top},
$$

with $\hat{\phi}_{1}$ and $\hat{\phi}_{\eta+1}$ known from the system's boundary conditions. This system is solved with a Thomas algorithm to compute the concentration $\hat{\phi}_{i}$ at the $i$-th interface $(i=2, \ldots, \eta)$.

Once the concentrations $\hat{\phi}$ at each interface have been computed in Laplace space, they are used as the boundary conditions for the single layer equations. This generates the solution for the concentration anywhere in the system. The solution is then numerically inverted using the de Hoog algorithm [4] (unless the final-value theorem is applied).

Herein lies the main advantage of the Laplace transform method. It allows the interface concentrations to be isolated as a multiple of a term in (26) rather than as a part of an integral inside of a term in (26). The isolation of the interface concentration allows each solution to be linked to the next as a series of linear equations. Thus making the solution easily solvable by use of an iterative solver.

\section{Results}

Numerous previous investigations have focused on cases with constant boundary conditions. Keeping in spirit with tradition, constant boundary conditions are supplied for Cartesian and spherical cases. A zero flux case is presented for the cylindrical coordinates. Periodic boundary conditions and reaction cases are presented to exemplify the versatility of this method. It should again be noted that steady-state solutions can only be found for boundary conditions that decay or grow to a constant value as time goes to infinity.

\subsection{Constant boundary conditions, no reactions}

In this example, the boundary conditions at the system's inlet and outlet are set to 1 and 0 , respectively. The coordinate systems for each layer in the three cases considered are Cartesian, cylindrical and spherical.

Figure 2 shows the Cartesian case with three Dirichlet bounded layers, each layer has equal 
thickness or length 1 , and zero initial concentration. The ratio of diffusion coefficients in the three layers is 1.0:0.2:0.4.

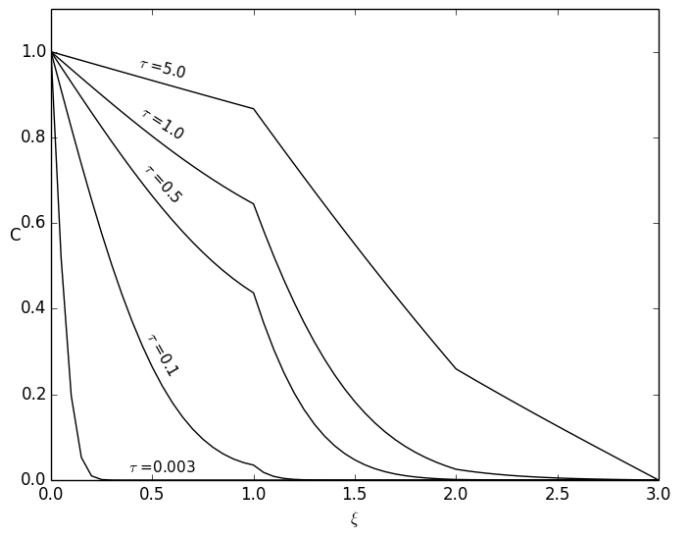

Figure 2: Dimensionless three-layer composite medium in the Cartesian coordinates with the Solid (Dirichlet)hollow-hollow case geometry. The layer interfaces are at $\xi=1.0$ and $\xi=2.0$ with all layers confined to $0 \leq \xi \leq 3$. The Damköhler number and dimensionless diffusion coefficient for each layer are $D a=0$ and $D_{c}=[1.0,0.2,0.4]$. The inlet and outlet dimensionless concentrations are $C_{i n}=1.0$ and $C_{\text {out }}=0.0$.

Figure 3 depicts the cylindrical case with a Neumann-Dirichlet layer with a no flux boundary condition at the origin and an initial concentration of 1.0 connected to a hollow layer bounded by a semi-infinite layer. The first two layers have a thickness of 1.0, and the ratio of diffusion coefficients is 1.0:0.2:0.4.

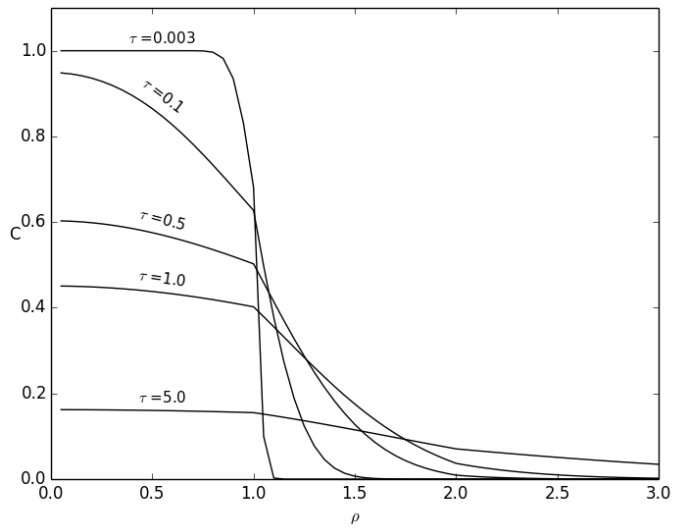

Figure 3: Dimensionless three-layer composite medium in the cylindrical coordinates with the Solid (Neumann)hollow-semi infinite case geometry. The layer interfaces are at $\rho=1.0$ and $\rho=2.0$ with all layers confined to $0 \leq \xi \leq \infty$. The initial concentration for the first layer in the region $0 \leq \xi \leq 1$ is $C_{0}=1.0$. The Damköhler number and dimensionless diffusion coefficient for each layer are $D a=0$ and $D_{c}=[1.0,0.2,0.4]$.

Figure 4 illustrates the spherical case away from the origin with two Dirichlet bounded layers connected to a semi infinite layer. Again the finite layer thicknesses are 1.0 and the ratio of diffusion coefficients is 1.0:0.2:0.4 with zero initial concentration.

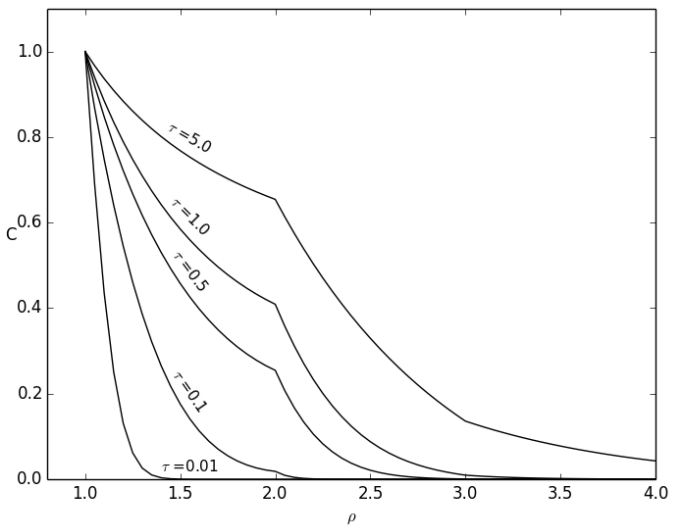

Figure 4: Dimensionless three layer composite media in Spherical coordinates with the Hollow (Dirichlet)-hollowsemi infinite case geometry. The layer interfaces are at $\rho=2$ and $\rho=3$ with all layers confined to $1 \leq \xi \leq \infty$. The Damköhler number and dimensionless diffusion coefficient for each layer are $D a=0$ and $D_{c}=[1.0,0.2,0.4]$. The inlet dimensionless concentration is $C_{i n}=1.0$.

\subsection{First-Order Reaction, Constant Boundaries}

The following two figures are in the Cartesian coordinate system and have the same properties and geometries as figure 2. However, this time a constant reaction coefficient has been added to each layer. The reaction coefficient is the same for each layer thus the ratio of the diffusion coefficient to the reaction coefficient is 1.0:.2.0.4.0. Figure 5 has a positive reaction coefficient and is thus increasing concentration by reaction. The solution from Figure 2 without reaction kinetics $(D a=0)$ is shown in Figures 5 and 6 as the dashed lines for reference. 


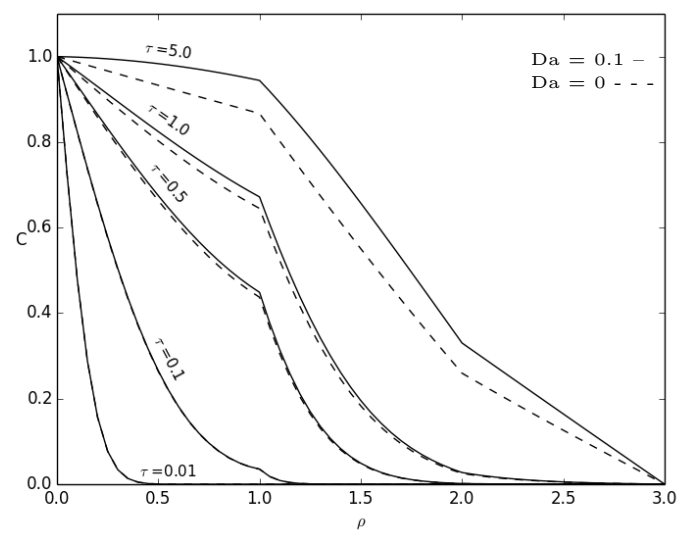

Figure 5: Dimensionless three-layer composite media in Cartesian coordinates with the Solid (Dirichlet)-hollowhollow case geometry. The layer interfaces are at $\xi=1$ and $\xi=2$ with all layers confined to $0 \leq \xi \leq 3$. The Damköhler number, dimensionless reaction coefficient, and dimensionless diffusion coefficient are $D a=0.1$, $R_{c}=[0.1,0.1,0.1]$ and $D_{c}=[1.0,0.2,0.4]$ respectively. The inlet and outlet dimensionless concentrations are $C_{\text {in }}=1.0$ and $C_{\text {out }}=0$.

Figure 6 has a negative reaction coefficient and is decreasing concentration by reaction.

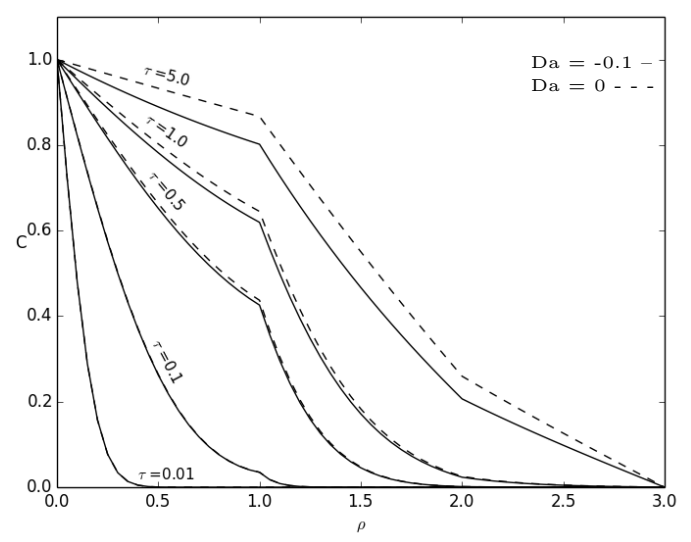

Figure 6: Dimensionless three-layer composite media in the Cartesian coordinates with the Solid(Dirichlet)hollow-hollow case geometry. The layer interfaces are at $\xi=1$ and $\xi=2$ with all layers confined to $0 \leq \xi \leq 3$. The Damköhler number, dimensionless reaction coefficient, and dimensionless diffusion coefficient are $D a=-0.1$, $R_{c}=[-0.1,-0.1,-0.1]$ and $D_{c}=[1.0,0.2,0.4]$ respectively. The inlet and outlet dimensionless concentrations are $C_{\text {in }}=1.0$ and $C_{\text {out }}=0$.

\subsection{Verification with Analytical Model}

The semi-closed solution is compared to the exact solution for the semi-infinite Cartesian single layer case. For simplicity, we impose a constant boundary condition, zero initial condition and no source term $\left(\phi_{a}=C_{i n}, C_{0}=0\right.$, and $\psi=0)$. Then, the Laplace-transformed concen- tration, $\hat{C}(\xi, s)$, is

$$
\hat{C}=\left(\hat{\phi}_{a}-\frac{C_{0}}{\mathcal{S}}\right) \mathcal{E}(-\xi)+\frac{C_{0}}{\mathcal{S}}=\frac{C_{i n}}{s} \mathcal{E}(-\xi) .
$$

Using the inverse Laplace transform

$$
\begin{aligned}
\mathcal{L}^{-1}\left(\frac{e^{-\alpha \sqrt{s+\beta^{2}}}}{s}\right) & =\frac{e^{-\alpha \beta}}{2} \operatorname{erfc}\left(\frac{\alpha}{2 \sqrt{\tau}}-\beta \sqrt{\tau}\right) \\
& +\frac{e^{\alpha \beta}}{2} \operatorname{erfc}\left(\frac{\alpha}{2 \sqrt{\tau}}+\beta \sqrt{\tau}\right)
\end{aligned}
$$

yields the exact solution, $C(\xi, \tau)$,

$$
\begin{aligned}
C & =\frac{C_{i n}}{2}\left[e^{(a-\xi) i \sqrt{\frac{D a R}{D}}} \operatorname{erfc}\left(\alpha_{\mathrm{ex}}-i \sqrt{D a R \tau}\right)\right. \\
& \left.+e^{(\xi-a) i \sqrt{\frac{D a R}{D}}} \operatorname{erfc}\left(\alpha_{\mathrm{ex}}+i \sqrt{D a R \tau}\right)\right] \cdot
\end{aligned}
$$

where $\alpha_{\mathrm{ex}}=(\xi-a) / \sqrt{4 D \tau}$.

Figures 7 and 8 show the comparison of the model with the exact solution for a hollow semi infinite piece. The reaction coefficient here is neglected. The geometry of the composite case compared to the semi infinite exact solution in Figures 7 and 8 is 5 Dirichlet bounded layers with the same material properties connected to a semi infinite layer. The first layer is offset from the origin to show that shifting the solution in space doesn't cause any errors. A diffusion reaction ratio of 100.0:1.0 is introduced in Figure 8.

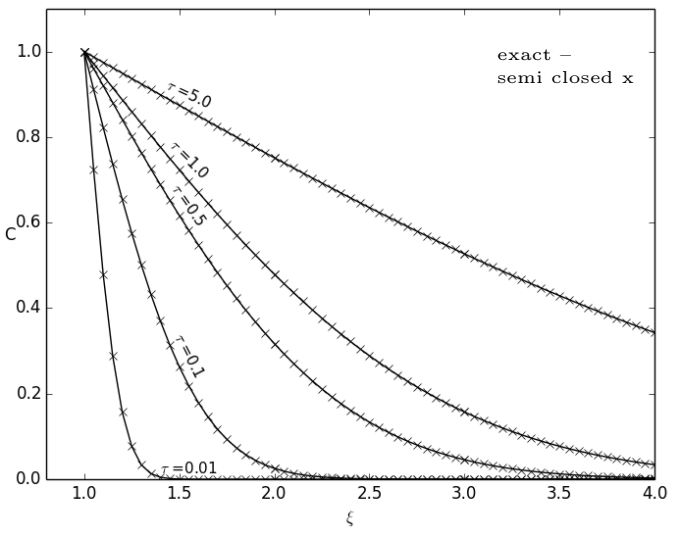

Figure 7: Dimensionless three-layer composite medium in Cartesian coordinates with the Hollow (Dirichlet)-hollowsemi infinite case geometry compared to a single semi infinite layer. The layer interfaces are at $\xi=2$ and $\xi=3$ with all layers confined to $1 \leq \xi \leq \infty$. The Damköhler number, dimensionless reaction coefficient, and dimensionless diffusion coefficient are $D a=0, R_{c}=0$ and $D_{c}=1.0$, respectively. The inlet dimensionless concentration is $C_{i n}=1.0$.

The relative error in the above comparison is less than $0.0001 \%$ even for large times. However, it can be seen in the Figure 8 that the introduction of the reaction coefficient can cause 
significant errors as time becomes large. This error is largely due to instabilities in the numerical Laplace transform method from numerical round off during calculation. As the time variable becomes large the corresponding Laplace variable becomes very small. For small time values the ratio of the Laplace variable to the reaction coefficient is large and errors are minimal. However as time becomes large the ratio becomes very small, eventually the reaction coefficient can mask the Laplace variable in some terms which causes the increasing error.

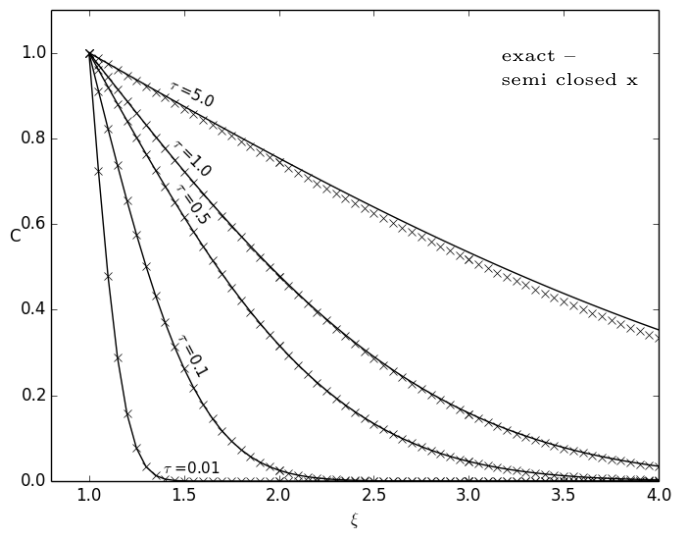

Figure 8: Dimensionless three-layer composite medium in Cartesian coordinates with the Hollow (Dirichlet)-hollowsemi infinite case geometry compared to a single semi infinite layer. The layer interfaces are at $\xi=2$ and $\xi=3$ with all layers confined to $1 \leq \xi<\infty$. The Damköhler number, dimensionless reaction coefficient, and dimensionless diffusion coefficient are $D a=-0.01, R_{c}=-0.01$ and $D_{c}=1.0$ respectively. The inlet dimensionless concentration is $C_{i n}=1.0$.

Figure 9 illustrates how the error in the model increases as time progresses. Again this is due to numerical round off. At $\tau=5.0$ the analytical model displays an error of $1.8 \%$. This error increases as $\tau$ and $D a$ increase. This error can be overcome by extending the floating point operations during numerical inversion.

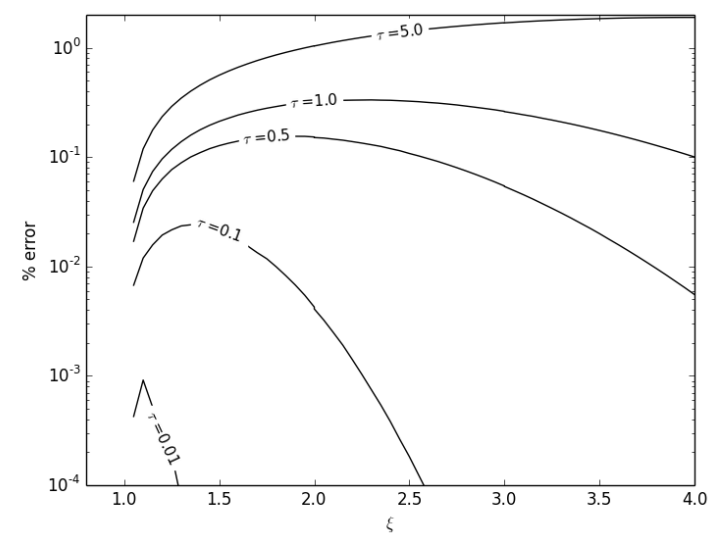

Figure 9: Relative error for the dimensionless three-layer composite medium in Cartesian coordinates with the Hollow (Dirichlet)-hollow-semi infinite case geometry compared to a single semi infinite layer. The layer interfaces are at $\xi=1$ and $\xi=2$ with all layers confined to $1 \leq \xi \leq \infty$. The Damköhler number, dimensionless reaction coefficient, and dimensionless diffusion coefficient are $D a=0, R_{c}=0$ and $D_{c}=1.0$ respectively. The inlet dimensionless concentration is $C_{i n}=1.0$.

\subsection{Transient Boundary Conditions}

As has been previously mentioned this method is capable of handling time dependent boundary conditions. Figure 10 demonstrates a case with the sinusoidal inlet concentration defined as $\phi_{1}=\alpha \sin (\nu \tau)+\gamma$ where $\alpha=1$ and $\gamma=0$ and $\nu=3$. In this case all layers are of the same thickness (1) and material properties.

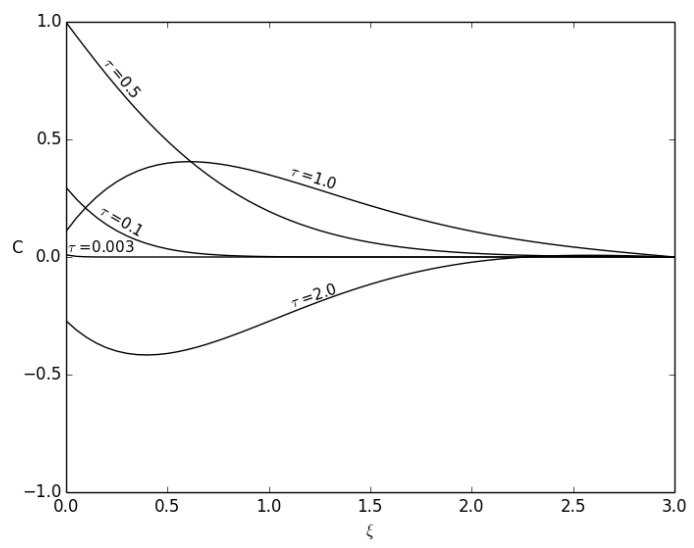

Figure 10: Dimensionless three-layer composite medium in Cartesian coordinates with the Solid (Dirichlet)-hollowhollow case geometry with a sinusoidal boundary condition defined by $\alpha \sin (\nu \tau)$. The layer interfaces are at $\xi=1$ and $\xi=2$ with all layers confined to $0 \leq \xi \leq 3$. The Damköhler number, dimensionless reaction coefficient, and dimensionless diffusion coefficient are $D a=-0.01$, $R_{c}=-0.01$ and $D_{c}=1.0$ respectively. The inlet and outlet dimensionless concentrations are $C_{i n}=\sin (3 \tau)$ and $C_{\text {out }}=0$ respectively.

Though the sinusoidal boundary condition is applicable in many scenarios it is subject to the same errors that emerge with the reaction solutions. When $\beta$ is large in comparison to the 
Laplace variable the boundary condition will tend toward a constant value. Thus it is recommended to only use periodic functions when the value of $\beta$ is small. For non periodic transient boundary conditions, however, this error may not emerge. Figure 11 depicts and example with the transient boundary condition $\phi_{1}=\tau^{1 / 8}$ with the same geometry as Figure 6.

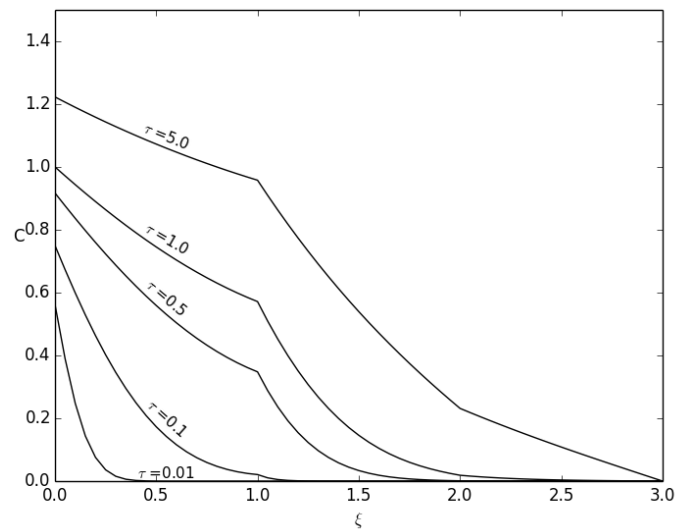

Figure 11: Dimensionless three-layer composite medium in Cartesian coordinates with the Solid (Dirichlet)-hollowhollow case geometry with an exponentially growing boundary condition defined by $\alpha \tau^{\nu}$. The layer interfaces are at $\xi=1$ and $\xi=2$ with all layers confined to $0 \leq \xi \leq 3$. The Damköhler number, dimensionless reaction coefficient, and dimensionless diffusion coefficient are $D a=-0.01, R_{c}=[-0.01,-0.01,-0.01]$ and $D_{c}=[1.0,0.2,0.4]$ respectively. The inlet and outlet dimensionless concentrations are $C_{i n}=\tau^{1 / 8}$ and $C_{\text {out }}=0$.

The key in avoiding numerical round off error in the inverse Laplace transform algorithm is to avoid terms that add or subtract the Laplace variable by a constant (e.g., $s-R$ ) but instead to have boundary conditions that are a multiple of the Laplace transform (e.g., $s \beta$ ).

\section{Conclusions}

A comprehensive method for a semi-closed analytical solution for composite media subject to diffusion and reaction kinetics has been presented. The method is robust enough to handle transient boundary conditions and to be computed with minimal time costs. An evaluation of the error in the solutions has been given. The error that emerges is subject to the number of digits of precision available for calculations. The presented plots were creating using the Matlab language which uses IEEE 754 double precision numbers. This has been found to be very accurate for diffusion only cases with non-transient boundary conditions. However for periodic transient boundary conditions and large reaction coefficients it has been shown that a higher precision would yield far better results. The general framework for the solutions presented here have the advantages of being applicable for both large and small times, as well as being able to handle a wide variety of transient boundary conditions. Future work will seek to expand these solutions to 2 and 3-dimensional cases, as well as handle higher order reaction terms. Other issues that will hopefully be overcome in the future is reaction dominated solutions with a large Damköhler number.

\section{Acknowledgements}

The authors thank Anita Carrasco-Griego, LANL W-4 for funding this work and Dr. Cuauhtemoc Aviles-Ramos, LANL W-13 for his careful review of the original manuscript. DMT was supported in part by the National Science Foundation under grant DMS-1522799. Los Alamos National Laboratory is an equal opportunity employer operated by Los Alamos National Security, LLC for the US Department of Energy's NNSA.

[1] C. Aviles-Ramos, A. Haji-Sheikh, J. V. Beck, and K. J. Dowding. Estimation of thermophysical properties by the spectral method-Development and evaluation. J. Heat Transf., 123(1):24-30, 2000.

[2] J. V. Beck, K. D. Cole, A. Haji-Sheikh, and B. Litkouhi. Heat Conduction Using Green's Functions. Hemisphere Publishing Co., 1992.

[3] H. S. Carslaw and J. C. Jaeger. Conduction of Heat in Solids 2nd ed. Oxford University Press, 1959.

[4] F. R. de Hoog, J. H. Knight, and A. N. Stokes. An improved method for numerical inversion of laplace transforms. SIAM. J. Sci. Stat. Comput., 3:357-366, 1982.

[5] C. J. Dias. A method of recursive images to solve transient heat diffusion in multilayer materials. Int. J. Heat Mass Transfer, 85(6):1075-1083, 2015.

[6] S. C. Huang and Y. P. Chang. Heat conduction in unsteady, periodic, and steady states in laminated composites. J. Heat Transf., 102(4):742-748, 1980. 
[7] R. Li, H. Cheng, Y. Su, S.-W. Hwang, L. Yin, H. Tao, M. A. Brenckle, D.-H. Kim, F. G. Omenetto, J. A. Rogers, and Y. Huang. An analytical model of reactive diffusion for transient electronics. Adv. Funct. Mater., 23(24):3106-3114, 2013.

[8] A. Ruiz Martinez, D. Roubinet, and D. M. Tartakovsky. Analytical models of heat conduction in fractured rocks. J. Geophys. Res. Solid Earth, 119(1):83-98, 2014.

[9] F. De Monte. An analytic approach to the unsteady heat conduction processes in one-dimensional composite media. Int. J. Heat Mass Transfer, 45(6):1333-1343, 2002.

[10] Ozisik. Heat Conduction. John Wiley \& Sons, Inc., 1980.

[11] Y. Sun and I. S. Wichman. On transient heat conduction in a one-dimensional composite slab. Int. J. Heat Mass Transfer, 47(6):1555-1559, 2004. 